\title{
Further Result of $\boldsymbol{H}$-Supermagic Labeling for Comb Product of Graphs
}

\author{
Ganesha Lapenangga P. , Aryanto, Meksianis Z. Ndii
}

University of Nusa Cendana

${ }^{*}$ Corresponding author. Email: ganesha.lapenangga@staf.undana.ac.id

\begin{abstract}
Let $G=(V, E)$ and $H=\left(V^{\prime}, E^{\prime}\right)$ be a connected graph. $H$-magic labeling of graph $G$ is a bijective function $f: V(G) \cup$ $E(G) \rightarrow\{1,2, \ldots,|V(G)|+|E(G)|\}$ such that for every subgraph $H^{\prime}$ of $G$ isomorphic to $H, \sum_{v \in V(H)} f(v)+$ $\sum_{e \in E(H)} f(e)=k$. Moreover, it is $H$-supermagic labeling if $f(V)=\{1,2, \ldots,|V|\}$. A graph $G$ having such labeling called $H$-supermagic graph. Next, we introduce comb product of graph. Suppose $G$ and $H$ are two connected graph and $o$ is vertex in $H$. A comb product between $G$ and $H$, denoted by $G \triangleright_{o} H$, is a graph obtained by taking a copy of graph $G$ and $|V(G)|$ copies of graph $H$, then identifying the $i$-th copy of graph $H$ at vertex $o$ to $i$-th vertex of graph $G$. In this paper, we construct $H_{1} \triangleright_{o} H_{2}$-supermagic labeling of graph $G \triangleright_{o} H_{2}$ where $G$ is $H_{1}$-supermagic graph.
\end{abstract}

Keywords: Comb product, H-supermagic labeling, H-magic.

\section{INTRODUCTION}

A graph $G$, denoted by $G=(V, E)$, is an ordered set consist of a non-vacuous set $V$ and a set $E \subseteq[V]^{2}$. We use $V(G)$ and $E(G)$ respectively as a notation of vertex and edge set of graph $G$ [1]. One of a branch theory in graph is graph labeling. There are so many types of labeling of graph, we can see it all in [2], but our focus in this paper related to $H$-magic labeling. This theory was introduced by Gutiérrez and Lladó [3]. Let us talk about formal definition of $H$-(super)magic graph and a requirement of it. Let $G$ and $H$ be a connected graph. A graph $G$ admits $H$-magic if for every edge $e \in E(G)$, there exist a subgraph $H^{\prime}$ in $G$ isomorphic to $H$ such that $e \in E\left(H^{\prime}\right)$ and there is a bijective function $f: V(G) \cup$ $E(G) \rightarrow\{1,2, \ldots,|V(G)|+|E(G)|\}$ which for every subgraph isomorphic to $H$, the sum of every vertex and edge label is equal to a number, named $k$. This number is called the magic number. Futhermore, it is said to be $H$ supermagic if $f(V)=\{1,2, \ldots,|V(G)|\}$ [2]. In [3], Gutiérrez and Lladó constructed a star $K_{1, h}$-magic labeling of a bipartite graph $K_{m, n}$. They also give a path $P_{h}$-magic labeling of graph $P_{n}$ and cycle $C_{n}$. Llado and Moragas also proved that graph $W_{n}$ for odd $n \geq 5$ is $C_{3}$ supermagic [4]. Ngurah and others give a view results related to $C_{n}$-supermagic graphs and $P_{h}$-supermagic graph. They also provided the $C_{3}$ and $C_{4}$-supermagic graphs such as book $B_{n}$, ladder $P_{n} \times P_{2}$, fan $P_{n}+K_{1}$, triangle ladder $T L_{n}$, and grid $P_{m} \times P_{n}$ for some positive integers $m$ and $n$ [5]. Roswitha and others also continued to work on cycle supermagic labeling. They provided cycle supermagic labeling of generalized jahangir graph $\left(J_{k, s}\right)$, wheel graph for even $n$, and also complete bipartite graph $K_{2, n}$ [6]. Chithra and others introduced the $C_{m}$-E -supermagic labeling of generalized book $B_{n, m}$, Grid, $G_{n, m}$, and friendship graph $G F_{n}^{m}$ [7]. Sadariria and Susanti also proved $H$-supermagic labeling of some graphs with cycle, such as corona product between star and cycle, also book and cycle [8]. We try to observe some problems in $H$-supermagic labeling. By doing that, we find a problem related to comb product of graph that was introduced by Saputro and others. Suppose $G$ and $H$ be a graph and $o$ is the vertex in $H$. A comb product between $G$ and $H$, denoted by $G \triangleright_{o} H$, is a graf obtained by taking a copy of graph $G$ and $|V(G)|$ copies of graph $H$ then identifying the $i$-th copy of graph $H$ at vertex $o$ to the $i$-th vertex of graph $G$ [9]. Putra constructed $P_{2} \triangleright_{o} G$-supermagic labeling for comb product of path $\left(P_{n}\right)$ and cycle $\left(C_{n}\right)$ with any graph $G[10]$. Following of the result, we construct the $H_{1} \triangleright_{0} H_{2}$-supermagic labeling of graph $G \triangleright_{0} H_{2}$ provided that $G$ is $H_{1}$ supermagic graph. 


\section{MAIN RESULT}

Let us give the vertex and edge set of graph $G \triangleright_{o} H_{2}$. Suppose that,

$$
\begin{aligned}
& |V(G)|=a,|E(G)|=b \\
& \left|V\left(H_{1}\right)\right|=y,\left|E\left(H_{1}\right)\right|=z \\
& \left|V\left(H_{2}\right)\right|=c,\left|E\left(H_{2}\right)\right|=d
\end{aligned}
$$

then, the vertex and edge set is

$V\left(G \triangleright_{o} H_{2}\right)=\left\{v_{i} \mid 1 \leq i \leq a\right\} \cup\left\{v_{i, j} \mid 1 \leq i \leq a ; 1 \leq\right.$ $j \leq c-1\}$

$E\left(G \triangleright_{o} H_{2}\right)=\left\{e_{l} \mid 1 \leq l \leq b\right\} \cup\left\{e_{i, h} \mid 1 \leq i \leq a ; 1 \leq\right.$ $h \leq d\}$.

For $Z$ is a set of integer, we write $\sum Z$ to describe sum of every integer in $Z$ and $[a, b]=\{x \in \mathbb{Z} \mid a \leq x \leq b\}$. Let us now give the theorem about $H_{1} \triangleright_{o} H_{2}$ magic labeling of graph $G \triangleright_{o} H_{2}$.

Theorem 2.1. Suppose that $G$ is $H_{1}$-supermagic graph and $\mathrm{H}_{2}$ is connected graph such that the number of subgraph $H_{1}$ in $G$ is equal to the number of subgraph $H_{1} \triangleright_{o} H_{2}$ in $G \triangleright_{o} H_{2}$. If $|V(G)|$ odd, then $G \triangleright_{o} H_{2}$ is $H_{1} \triangleright_{o} H_{2}$-supermagic graph.

Proof. If $H_{2}$ is trivial graph [1], then $G \triangleright_{o} H_{2}=G$ and $H_{1} \triangleright_{o} H_{2}=H_{1}$, so it is clear by the premise. Next, suppose not. Since $G$ is $H_{1}$-supermagic graph, then there exist bijective function $f: V(G) \cup E(G) \rightarrow$ $\{1,2, \ldots,|V(G)|+|E(G)|\}$ such that $\sum_{v \in V(H)} f(v)+$ $\sum_{e \in E(H)} f(e)=k$ for all subgraph $H$ isomorphic to $H_{1}$ and $f(V)=\{1,2, \ldots,|V(G)|\}$. Assume that $s(f)=$ $\{f(v) \mid v \in V(H)\} \cup\{f(e) \mid e \in E(H)\}$ is an arbitrary set of vertex and edge label of subgraph $H$ isomorphic to $H_{1}$. We get that $\sum s(f)=k$. Then, define a function $g$ as a label to graph $G \triangleright_{o} H_{2}$. We divide it in two cases.

Case 1. $c+d$ even

Note that since $\mathrm{H}_{2}$ is not a trivial graph, then $c \geq 3$ for odd $c$ and $c \geq 4$ for even $c$. Then, define a function

$$
\begin{aligned}
& g\left(v_{i}\right)=f\left(v_{i}\right) ; 1 \leq i \leq a \\
& g\left(v_{i, 1}\right)= \begin{cases}\frac{(3 a+3)}{2}-i, i \in\left[1, \frac{a+1}{2}\right] \\
\frac{(5 a+3)}{2}-i, i \in\left[\frac{a+3}{2}, a\right]\end{cases} \\
& g\left(v_{i, 2}\right)= \begin{cases}2 a+2 i-1, & i \in\left[1, \frac{a+1}{2}\right] \\
a+2 i-1, & i \in\left[\frac{a+3}{2}, a\right]\end{cases} \\
& g\left(v_{i, j}\right)=\left\{\begin{array}{ll}
j a+a+1-i ; i \in[1, a], \text { odd } j \in[3, c-1] \\
j a+i ;
\end{array} \quad i \in[1, a], \text { even } j \in[3, c-1]\right.
\end{aligned}
$$

$$
g\left(e_{i, h}\right)=\left\{\begin{array}{l}
(c+h) a+1-i ; i \in[1, a], \text { odd } h \in[1, d] \\
(c+h-1) a+i ; i \in[1, a] \text { even } h \in[1, d]
\end{array}\right.
$$

$g\left(e_{l}\right)=(c+d-1) a+f\left(e_{l}\right) ; 1 \leq l \leq b$

It is clear that the function is a bijective. Note that if we sum all label of vertex and edge from an arbitrary subgraph $\mathrm{H}_{2}$, we get that

$$
\begin{aligned}
\sum_{v \in V\left(H_{2}\right) \backslash\{0\}} g(v)+ & \sum_{e \in E\left(H_{2}\right)} g(e) \\
& =\frac{a c^{2}+a d^{2}+c+d-a-1}{2}+a c d
\end{aligned}
$$

Let $\quad s(g)=\left\{g(v) \mid v \in V\left(H_{1} \triangleright_{o} H_{2}\right)\right\} \cup\{g(e) \mid e \in$ $\left.E\left(H_{1} \triangleright_{o} H_{2}\right)\right\}$. According to the function defined at Equation (2)-(6),

$$
\begin{aligned}
\sum s(g)= & k+a c z+a d z-a z+a c d y+ \\
& \frac{a c^{2} y+a d^{2} y+c y+d y-a y-y}{2}
\end{aligned}
$$

It means that the sum is equal for any subgraph isomorphic to $H_{1} \triangleright_{o} H_{2}$.

Case 2. $c+d$ odd

Define a function

$g\left(v_{i}\right)=f\left(v_{i}\right) ; 1 \leq i \leq a$

$g\left(v_{i, j}\right)=\left\{\begin{array}{l}j a+a+1-i ; i \in[1, a], \text { odd } j \in[1, c-1] \\ j a+i ; \quad i \in[1, a], \text { even } j \in[1, c-1]\end{array}\right.$

$g\left(e_{i, h}\right)=\left\{\begin{array}{l}c a+(h-1) a+i ; i \in[1, a], \text { odd } h \in[1, d] \\ c a+h a+1-i ; i \in[1, a], \text { even } h \in[1, d]\end{array}\right.$

$g\left(e_{l}\right)=(c+d-1) a+f\left(e_{l}\right) ; 1 \leq l \leq b$

Then, by the same method we apply in Case 1, we get that

$$
\begin{aligned}
\sum s(g)= & k+a c z+a d z-a z+a c d y+ \\
& \frac{a c^{2} y+a d^{2} y+c y+d y-a y-y}{2}
\end{aligned}
$$

From the both cases above, we get that $g\left(V\left(G \triangleright_{o} H_{2}\right)\right)=\{1,2, \ldots, a c\} \quad$ where $a c=$ $\left|V\left(G \triangleright_{o} H_{2}\right)\right|, g$ is a bijective function, and for all subgraph of $G \triangleright_{o} H_{2}$ isomorphic to $H_{1} \triangleright_{o} H_{2}$, the sum of every vertex and edge label is constant. So, we conclude that $g$ is $H_{1} \triangleright_{o} H_{2}$-supermagic labeling of graph $G \triangleright_{o} H_{2}$ where $|V(G)|$ is odd.

Note that this is not just hold for odd $|V(G)|$, but it also hold for even $|V(G)|$ and odd $\left|V\left(H_{2}\right)\right|+\left|E\left(H_{2}\right)\right|$. The proof is similar to the Theorem 2.1 , by taking a function defined in Case 2, then apply to the proof. By the short explanation, we derive it into this corollary. 
Corollary 2.2. Suppose that $G$ is $H_{1}$-supermagic graph and $\mathrm{H}_{2}$ is connected graph such that the number of subgraph $H_{1}$ in $G$ is equal to the number of subgraph $H_{1} \triangleright_{o} H_{2}$ in $G \triangleright_{o} H_{2}$. If $|V(G)|$ is even and $\left|V\left(H_{2}\right)\right|+$ $\left|E\left(H_{2}\right)\right|$ odd, then $G \triangleright_{o} H_{2}$ is $H_{1} \triangleright_{o} H_{2}$-supermagic graph.

Here is an example. In [6], Roswitha and others give a $C_{3}$-supermagic labeling of graph $W_{4}$ with magic number $k=36$. By using the Theorem 2.1 and adding comb product to $W_{4}$ with star $K_{1,3}$, we can create $C_{3} \triangleright_{0} K_{1,3}$ supermagic labeling of graph $W_{4} \triangleright_{0} K_{1,3}$. We provide it in Figure 1.

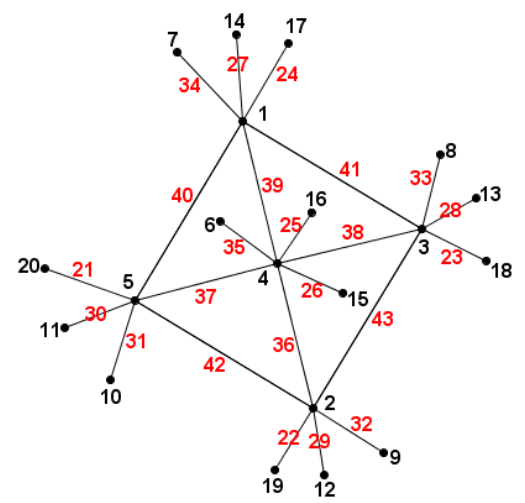

Figure 1 The $C_{3} \triangleright_{0} K_{1,3}$-supermagic labeling of graph $W_{4} \triangleright_{o} K_{1,3}$

Note that the magic number is $k^{*}=495$. We can get that by substituting $a=5, c=4, d=y=z=3, k=36$ to $k^{*}=k+a c z+a d z-a z+a c d y+$ $\frac{a c^{2} y+a d^{2} y+c y+d y-a y-y}{2}$. We also give an open problem for even $|V(G)|$ and even $\left|V\left(H_{2}\right)\right|+\left|E\left(H_{2}\right)\right|$.

Open Problem 1. For even $|V(G)|$ and even $\left|V\left(H_{2}\right)\right|+$ $\left|E\left(H_{2}\right)\right|$, where the number of subgraph $H_{1}$ in $G$ is equal to the number of subgraph $H_{1} \triangleright_{o} H_{2}$ in $G \triangleright_{o} H_{2}$, determine the $H_{1} \triangleright_{0} H_{2}$-supermagic labeling of $G \triangleright_{o} H_{2}$.

\section{CONCLUSION}

For any graph $G, H_{1}, H_{2}$, odd $|V(G)|$, the graph $G \triangleright_{o} H_{2}$ is $H_{1} \triangleright_{o} H_{2}$-supermagic with constant $k^{*}=k+a c z+$ $a d z-a z+a c d y+\frac{a c^{2} y+a d^{2} y+c y+d y-a y-y}{2}$ for $a, c, d$ in Equation (1) and $k$ is the magic number of $H_{1}$ supermagic labeling of graph $G$. Moreover, for even $|V(G)|$ and odd $\left|V\left(H_{2}\right)\right|+\left|E\left(H_{2}\right)\right|$, the graph $G \triangleright_{o} H_{2}$ is $H_{1} \triangleright_{o} H_{2}$-supermagic with same constant above.

\section{ACKNOWLEDGMENTS}

The first author was funded by "Hibah Fakultas" Research Grant

No.

003/UN15.15.2.PPK/SPP/FST/IV/2021 from Faculty of Science and Technology University of Nusa Cendana.

\section{REFERENCES}

[1] R. Diestel, Graph Theory (5 ${ }^{\text {th }}$ Ed.), Berlin, Springer, 2017.

[2] J. A. Galian, A dynamic survey of graph labelling, Electronic Journal of combinatorics, 2018, $23^{\text {rd }}$ Ed., Dynamic Surveys, \#DS6.

[3] A. Gutiérrez, A. Lladó, Magic coverings, J. Combin. Math. Combin. Comput., Vol. 55, 2005, pp. 43-56.

[4] A. Lladó, J. Moragas, Cycle-magic graphs, Discrete Math., vol. 307, 2007, pp. 2925-2933. DOI: https://doi.org/10.1016/j.disc.2007.03.007.

[5] A. A. G Ngurah, A. N. M Salman, L. Susilowati, $H$ supermagic labelings of graphs, Discrete Mathematics, 2010, pp. 1293-1300. DOI: https://doi.org/10.1016/j.disc.2009.12.011.

[6] M. Roswitha, E. T. Baskoro, T. K. Maryati, N. A. Kurdhi \& I. Susanti, Further results on cyclesupermagic labeling, AKCE International Journal of Graphs and Combinatorics, Vol. 10(2), 2013, pp. 211-220,

DOI: https://doi.org/10.1080/09728600.2013.12088737.

[7] C. Chithra, G. Marimuthu, G. Kumar. $C_{m}-E$ supermagic labeling of graphs. AKCE International Journal of Graphs and Combinatorics, Vol. 17(1), 2019, pp. 510-518 DOI: https://doi.org/10.1016/j.akcej.2019.03.016.

[8] H. Sadariria, Y. Susanti, $H$-supermagic labeling on edge coronation of some graphs with a cycle, AIP Conference Proceedings, AIP Publishing LLC, 2192, 2019, pp. 040014, DOI: https://doi.org/10.1063/1.5139140.

[9] S. Wido Saputro, N. Mardiana, I. A. Purwasih, The metric Dimension of Comb Product Graphs, MATEMATIK VESNIK Vol. 69(4), 2017, pp. 248258.

[10]G. L. Putra, The $P_{2} \triangleright_{o} G$-supermagic labeling of comb product of graphs, Tensor: Pure and Applied Mathematics Journal, Vol. 2(1), 2021, pp. 13-18. 